\title{
First light measurements with the XMM-Newton reflection grating spectrometers: Evidence for an inverse first ionisation potential effect and anomalous Ne abundance in the Coronae of HR 1099
}

\author{
A. C. Brinkman ${ }^{1}$, E. Behar ${ }^{2}$, M. Güdel ${ }^{3}$, M. Audard ${ }^{3}$, A. J. F. den Boggende ${ }^{1}$, G. Branduardi-Raymont ${ }^{4}$, \\ J. Cottam ${ }^{2}$, C. $\operatorname{Erd}^{5}$, J. W. den Herder ${ }^{1}$, F. Jansen ${ }^{5}$, J. S. Kaastra ${ }^{1}$, S. M. Kahn², R. Mewe ${ }^{1}$, \\ F. B. S. Paerels ${ }^{2}$, J. R. Peterson ${ }^{2}$, A. P. Rasmussen ${ }^{2}$, I. Sakelliou ${ }^{4}$, and C. de Vries ${ }^{1}$ \\ 1 Space Research Organization of The Netherlands, Sorbonnelaan 2, 3584 CA Utrecht, The Netherlands \\ 2 Columbia Astrophysics Laboratory, Columbia University, 550 West 120th Street, New York, NY 10027, USA \\ 3 Paul Scherrer Institute, Würenlingen and Villigen, 5235, Switzerland \\ 4 Mullard Space Science Laboratory, University College London, Dorking RH5 6NS, UK \\ 5 Astrophysics Division, Space Science Department of ESA, ESTEC, 2200 AG Noordwijk, The Netherlands
}

Received 2 October 2000 / Accepted 24 October 2000

\begin{abstract}
The RSCVn binary system HR 1099 was extensively observed by the XMM-Newton observatory in February 2000 as its first-light target. A total of $570 \mathrm{ks}$ of exposure time was accumulated with the Reflection Grating Spectrometers (RGS). The integrated X-ray spectrum between $5-35 \AA$ is of unprecedented quality and shows numerous features attributed to transitions of the elements $\mathrm{C}, \mathrm{N}, \mathrm{O}, \mathrm{Ne}, \mathrm{Mg}, \mathrm{Si}, \mathrm{S}, \mathrm{Fe}$, and Ni. We perform an in-depth study of the elemental composition of the average corona of this system, and find that the elemental abundances strongly depend on the first ionisation potential (FIP) of the elements. But different from the solar coronal case, we find an inverse FIP effect, i.e., the abundances (relative to oxygen) increase with increasing FIP. Possible scenarios, e.g., selective enrichment due to Ne-rich flare-like events, are discussed.
\end{abstract}

Key words. atomic processes - line: formation - nuclear reactions, nucleosynthesis, abundances - stars: coronae stars: individual (HR 1099) - X-rays: stars

\section{Introduction}

The outer atmospheres of stars are of fundamental importance to the chemical enrichment of interstellar space through stellar winds and the ejection of energetic particles (Meyer 1985). Interestingly, the elemental composition of the hot magnetic solar corona and the solar wind are markedly different from the Sun's surface composition, which suggests that some fractionation process in the denser layers of the solar atmosphere must selectively enrich the corona with particular elements. Various observations of the solar corona, the solar wind, solar energetic particles, and cosmic rays have established that elements with a first ionisation potential (FIP) below $10 \mathrm{eV}$ are overabundant by factors of up to 4 with respect to the solar photosphere, unlike elements with a high FIP that maintain their photospheric abundance (Feldman 1992; see also Meyer 1985). This is usually referred to as the "FIP effect". However, the physics of this fractionation

Send offprint requests to: B. Brinkman mechanism is poorly understood. Comparative abundance studies of stars other than the Sun may shed light on the underlying physics.

The X-ray regime contains a rich forest of atomic spectral lines emitted by many chemical species at ionisation stages corresponding to plasma temperatures between $\sim 1$ and 30 Million K. So far, however, attempts to determine the composition of stellar coronae other than the Sun's from X-ray observations (Antunes et al. 1994; White 1996; Drake 1996) have produced results that are considered to be ambiguous, because the low spectral resolution available from even the best nondispersive detectors has not permitted the separation of atomic emission lines arising from different elements. Often, subphotospheric abundances were claimed. Studies of stellar coronal abundances with the EUVE satellite have revealed solar-like FIP effects in some, but not all of the stars studied (Drake et al. 1995, 1997). The high-resolution grating spectrometers on board the recently launched American Chandra and European $X M M-N e w t o n$ satellites now provide a new 


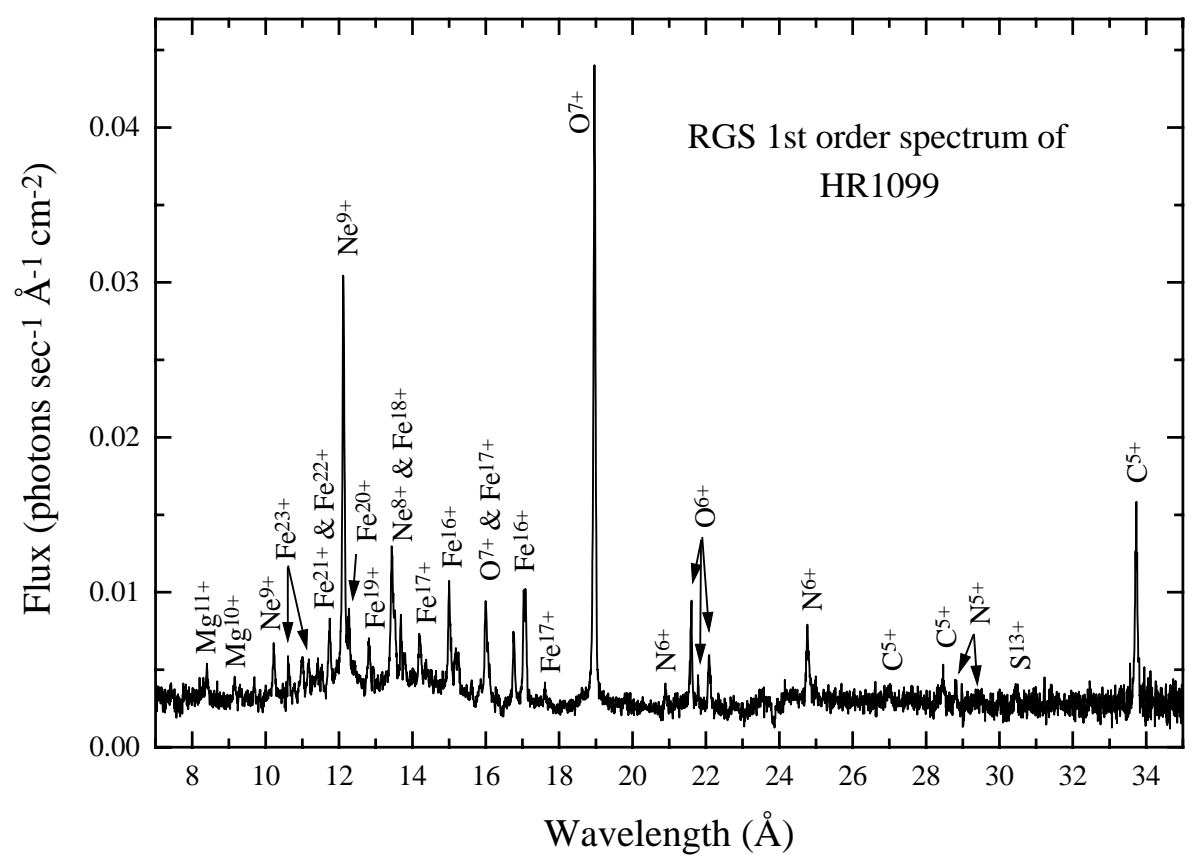

Fig. 1. First order spectrum of HR 1099 as measured by the RGS instruments on board XMM-Newton. The most prominent spectral features are indicated just above the spectrum by the corresponding emitting ion opportunity to unambiguously determine both the elemental abundances and the thermal structure of stellar coronae through explicit measurement of emission line and continuum intensities in the X-ray band. We are using grating spectroscopy on XMM-Newton to perform such analyses for the first time. The target chosen for the present observation is HR 1099, one of the X-ray brightest and most magnetically active stellar systems, which was observed early in the programme. HR 1099 has been the target of previous EUVE and low-resolution X-ray spectroscopic investigations (e.g. Pasquini et al. 1989; Griffith \& Jordan 1998). Ayres et al. (2001) qualitatively compare the Chandra HETG spectra of HR 1099 and Capella, the latter being a somewhat cooler and less active coronal source. A companion paper (Audard et al. 2001) to the present work discusses spectral variability in HR 1099, based on selected XMM-Newton RGS data sets.

\section{Observations and analysis}

The $X M M$-Newton X-ray observatory incorporates a payload with two identical high-resolution Reflection Grating Spectrometers (RGS; den Herder et al. 2001) with a spectral resolution of about $0.06 \AA F W H M$. The spectral band covered by the RGS ranges from 5 to $35 \AA$. This range contains strong lines of $\mathrm{C}, \mathrm{N}, \mathrm{O}, \mathrm{Ne}, \mathrm{Mg}, \mathrm{Si}, \mathrm{S}, \mathrm{Ca}, \mathrm{Fe}$, and Ni. The clearly detected eight consecutive charge states of Fe are of crucial importance for the present analysis. HR 1099 was observed in January and February 2000 for a total of $570 \mathrm{ksec}$ yielding approximately 1.3 million source counts. The spectrum was extracted by counting events inside a narrow spatial window along the dispersed CCD image, and then by using the CCD energy resolution to separate the spectral orders. The extracted total first-order spectrum between 7 and $35 \AA$ is shown in Fig. 1 .

\section{Results}

In optically thin coronal plasmas, the power of a particular spectral line depends on the collisional and radiative transition rates. In this work, these quantities are calculated by means of the relativistic Hebrew University Lawrence Livermore Atomic Code (Bar-Shalom et al. 1998). Owing to the high spectral resolution, we can directly scale the contribution of each ion species to match the observation. The atomic-level models are each calculated for a single electron temperature, $T_{\max }$, at which the relevant ion has its maximum abundance. Figure 2 shows the theoretical spectrum from $7-18 \AA$ (red curve), which also includes a phenomenological continuum component (blue) corresponding to a bremsstrahlung spectrum (which dominates over any other continuum components at the temperatures in question), compared with the HR 1099 data points (black). As can be seen, the agreement is very good. The majority of lines in this region are due to highly ionised $\mathrm{Fe}$ as demonstrated by the individual ion curves at the bottom of the figure. The contribution of each ion species yields a direct measurement of the amount of coronal gas in the conditions appropriate for that ion.

With the distance $d(=29 \mathrm{pc})$ to HR 1099 , the calculated line power and the measured line flux can be used to obtain the emission measure $E M$ (in $\mathrm{cm}^{-3}$ ), which is a product of the electron density and the H-ion density, integrated over the emitting volume. We assume that each ion emits mostly around the temperature $T_{\max }$. This leads to a first approximation of the emission measure distribution. This distribution is sufficiently accurate for our purposes if no steep gradients are present. Finally, in order to plot the $E M$, we use the ionic abundances from ionisation balance calculations (Arnaud \& Raymond 1992; Mazotta 1998) and assume, as a first step, elemental abundances of the solar photosphere. The resulting $E M$ as a function 


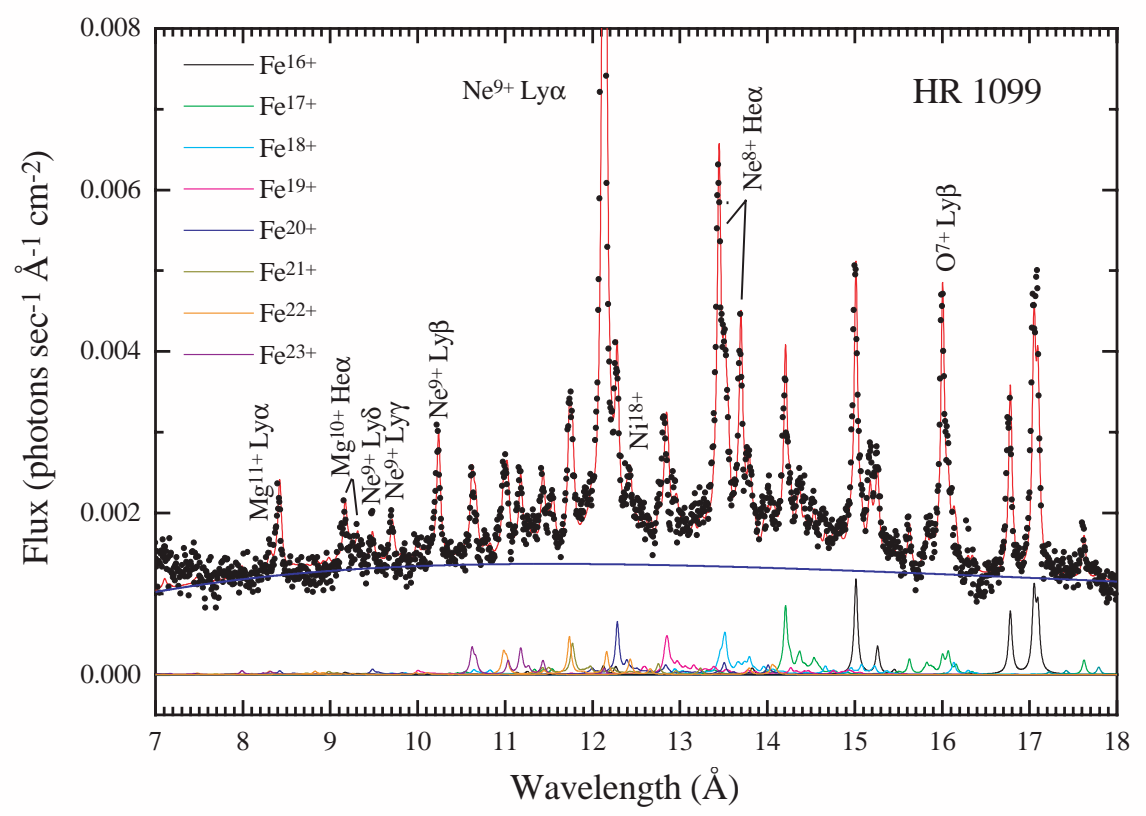

Fig. 2. Observed HR 1099 flux spectrum (black dots) compared with the total calculated spectrum (red curve). The inferred thermal bremsstrahlung continuum is represented by the blue curve. The eight separate plots at the bottom show the relative individual-ion contributions of $\mathrm{Fe}^{16+}$ to $\mathrm{Fe}^{23+}$ (not to scale). The non-Fe lines ( $\mathrm{O}, \mathrm{Ne}, \mathrm{Mg}$, and $\mathrm{Ni}$ ) are indicated explicitly

Table 1. Abundance estimates ${ }^{a}$ of the HR 1099 coronae, Solar photosphere $^{b}$ and Solar corona ${ }^{c}$ relative to O

\begin{tabular}{ccccccc}
\hline Element & $\begin{array}{c}\text { FIP } \\
(\mathrm{eV})\end{array}$ & $\begin{array}{c}\text { HR 1099 } \\
\text { coronae } \\
\text { (present) }\end{array}$ & $\begin{array}{c}\text { Solar } \\
\text { photo- } \\
\text { sphere }\end{array}$ & $\begin{array}{c}\text { Solar } \\
\text { corona }\end{array}$ & $\begin{array}{c}\text { Solar } \\
\text { corona/Solar } \\
\text { photosphere }\end{array}$ & $\begin{array}{c}\text { HR 1099 } \\
\text { coronae/Solar } \\
\text { photosphere }\end{array}$ \\
\hline $\mathrm{Ne}$ & 21.56 & 0.57 & 0.15 & 0.15 & 1.0 & 3.8 \\
$\mathrm{~N}$ & 14.53 & 0.17 & 0.12 & 0.13 & 1.08 & 1.4 \\
$\mathrm{O}$ & 13.61 & 1.0 & 1.0 & 1.0 & 1.0 & 1.0 \\
$\mathrm{C}$ & 11.26 & 0.28 & 0.47 & 0.50 & 1.06 & 0.59 \\
$\mathrm{~S}$ & 10.36 & 0.0085 & 0.019 & 0.024 & 1.26 & 0.45 \\
$\mathrm{Fe}$ & 7.87 & 0.0094 & 0.038 & 0.16 & 4.21 & 0.25 \\
$\mathrm{Mg}$ & 7.64 & 0.043 & 0.045 & 0.18 & 4.0 & 0.95 \\
$\mathrm{Ni}$ & 7.63 & 0.00070 & 0.0021 & 0.0089 & 4.24 & 0.33 \\
\hline${ }^{a}$ Error estimates are 20\% for all numbers. \\
${ }^{b}$ Feldman (1992). \\
${ }^{c}$ Feldman et al. (1992).
\end{tabular}

of the electron temperature $T_{\mathrm{e}}$ is shown in Fig. 3 for ions of $\mathrm{C}, \mathrm{N}, \mathrm{O}, \mathrm{Ne}, \mathrm{Mg}, \mathrm{S}, \mathrm{Fe}$, and $\mathrm{Ni}$. These ions sample the HR 1099 hot coronae in the $T_{\mathrm{e}}$ range from $k T_{\mathrm{e}}=100 \mathrm{eV}$ to $1500 \mathrm{eV}$ and illustrate the monotonic increase of the $E M$ in this range. The errors on the temperature for each ion represent the range of $T_{\mathrm{e}}$ for which the ion fraction exceeds $50 \%$ of its maximum value, except for the He-like ions $\mathrm{O}^{6+}, \mathrm{Ne}^{8+}$, and $\mathrm{Mg}^{10+}$, for which line ratio methods (Gabriel \& Jordan 1969) are used to determine $T_{\mathrm{e}}$ more precisely and the bars reflect a $25 \%$ uncertainty.

Looking only at the eight Fe data points in Fig. 3, the $E M$ distribution appears to be approximately a smooth power law over the relevant temperature range as indicated by the solid line. The use of Fe alone provides a representation of the $E M$ behaviour independent of elemental abundance. Note that the power-law $E M$ distribution obtained for Fe is strikingly parallel to the $E M$ trends suggested by the data points for $\mathrm{O}, \mathrm{Ne}$, and $\mathrm{Mg}$. However, the distribution is vertically offset for these other elements.
The corrections needed in order to settle these offsets allow us to estimate the abundances relative to one another. The high degree of overlap in the $T_{\mathrm{e}}$ ranges of formation, especially for the $\mathrm{Ne}, \mathrm{Mg}, \mathrm{Fe}$, and $\mathrm{Ni}$ ions, makes the sense of the relative abundance determinations unambiguous. Table 1 presents our derived approximate abundances with the solar photospheric and solar coronal abundances. The latter should be considered as average abundances in various solar coronal features in which the individual abundances may vary somewhat (Hénoux 1995). Similarly, our HR 1099 results are average coronal abundances during our observations. We note that we give abundances relative to the well-determined, abundant, and well-behaved (on the Sun) high-FIP element O. Relative abundances are appropriate for the study of possible dependencies on the FIP. They also avoid complications with the detailed modeling of the continuum. We plot in Fig. 4 the coronal enrichment (relative to solar photospheric abundances) for the HR 1099 coronae as a function of the FIP. 

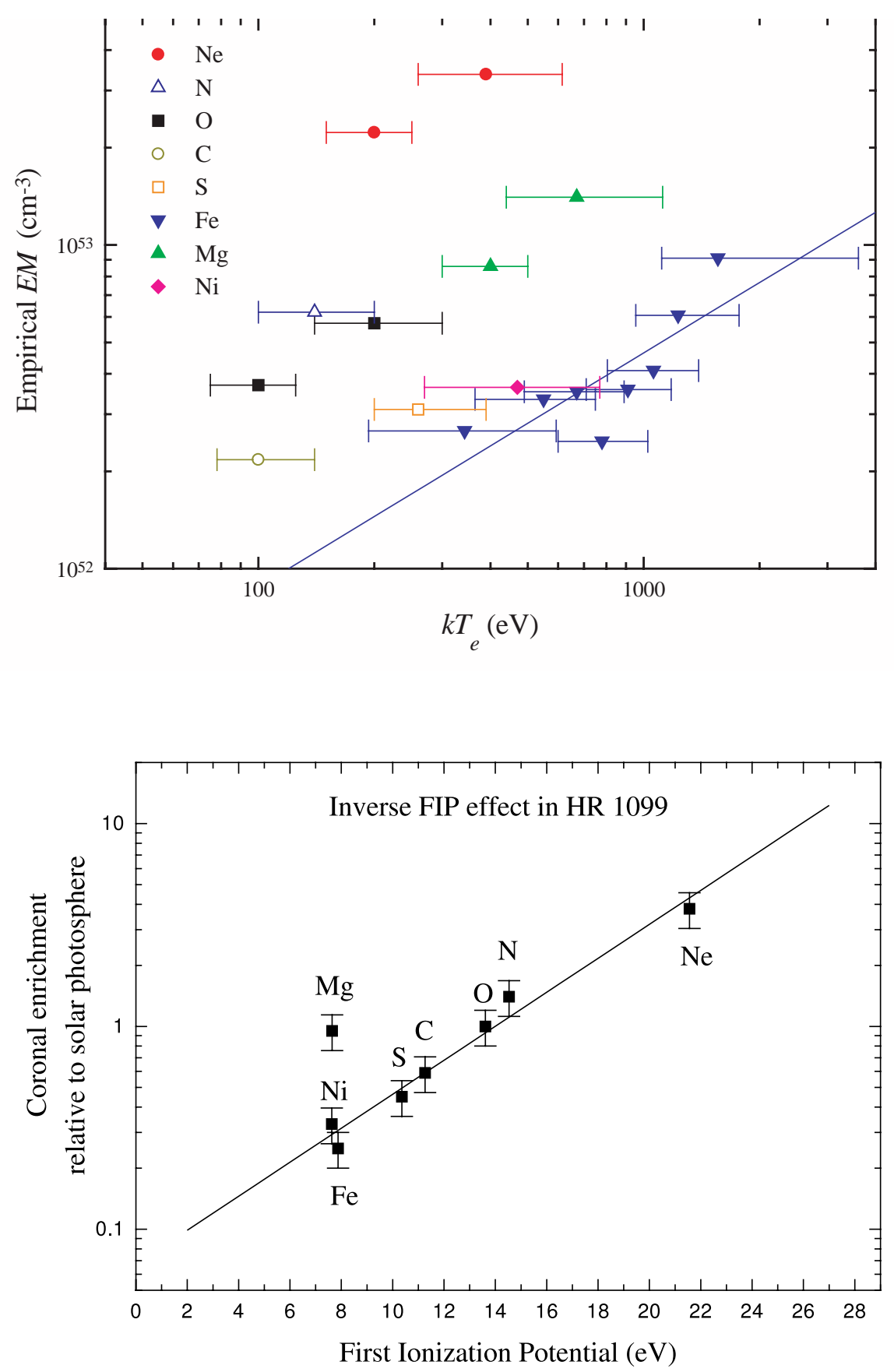

Fig. 3. The empirical emission measure for: $\mathrm{O}^{6+}, \mathrm{O}^{7+}, \mathrm{Ne}^{8+}, \mathrm{Ne}^{9+}, \mathrm{Mg}^{10+}$, $\mathrm{Mg}^{11+}, \mathrm{Fe}^{16+}$ through $\mathrm{Fe}^{23+}, \mathrm{Ni}^{18+}$, $\mathrm{C}^{5+}, \mathrm{N}^{6+}$ and $\mathrm{S}^{13+}$ (in order from left to right for each element). The straight line is the best-fit power-law for the Fe ions alone
Fig. 4. Enrichment of elemental abundances in the HR 1099 coronae relative to (solar) photospheric values, plotted as a function of the first ionisation potential (FIP). All values are given error bars of $20 \%$. Note the increase with FIP, exactly opposite the trend observed in the average solar corona
We note in passing that the precise composition of the HR 1099 photosphere (except for a recent measurement of the Fe abundance of both HR 1099 components, Randich et al. 1994) is unknown. It is expected that overall, the photospheres of nearby field stars such as HR 1099 have a mixture of elements similar to the Sun.

\section{Discussion}

The Ne/O ratio found here for the HR 1099 coronae is unusually high compared with the solar photospheric value, while the $\mathrm{N} / \mathrm{O}$ ratio is also enhanced and the $\mathrm{Mg} / \mathrm{O}$ ratio is close to solar photospheric. The abundance ratios for the remaining elements are well below their solar photospheric values. Compared with the respective ratios of the solar corona, the contrast is even sharper. In fact, HR 1099 reveals systematically a reversed FIP effect, which is illustrated in Fig. 4; the coronal enrichment increases monotonically with the FIP. The only exception may be $\mathrm{Mg}$ (low FIP). Ne is strongly enhanced, by a factor of $3.8 \mathrm{com}-$ pared to the solar photosphere. This enhancement is reminiscent of a similar anomaly observed in a subset of solar flares (Schmelz 1993; Murphy et al. 1991). Interestingly, flares are thought to be of great importance to the heating 
of apparently quiet coronae of active stars (Doyle \& Butler 1985; Güdel 1994; Audard et al. 2000) and have been suggested to be pivotal for the elemental fractionation process as well (Wang 1996; Güdel et al. 1999; Mewe et al. 1997). Continuous mixing of photospheric and coronal material in low-lying loops through frequent flares could suppress the solar-like FIP effect (Feldman \& Widing 1990) in these stars altogether.

It is generally believed that the fractionation is the result of diffusive processes across magnetic field lines somewhere in the chromosphere, where the ionisation fraction differs substantially among elements of high or low FIP (von Steiger \& Geiss 1989). Low FIP elements can then be accelerated into the quiescent corona, leaving an excess of high FIP elements in the chromosphere (Schmelz 1993). If this chromospheric layer can be accessed by flares (Schmelz 1993), then a high-FIP-rich plasma may be heated and lifted into dense coronal X-ray loops giving rise to the reversed coronal FIP effect observed in HR 1099. In any case, it is clear that the simple mechanism involving steady-state diffusion, which has been proposed for the quiet solar corona, does not operate in more active stars such as HR 1099. Since cosmic rays do show a low FIP enhancement, it is unlikely that such active stars can be major contributors to the cosmic ray flux. The wide survey of stellar coronae planned with the RGS on XMM-Newton will provide more general answers to the questions raised by these observations.

Acknowledgements. We thank the many teams in industry and at the scientific institutions and ESA, for building such an excellent spacecraft and payload - and operating it. We are particularly grateful to Mr. Robert Lainé and his project team at ESA for leading the project. We thank the XMM-Newton SOC staff for making the commissioning phase data available to us. SRON is financially supported by the Netherlands Organization for Scientific Research (NWO). The Columbia University team acknowledges generous support from the National Aeronautics and Space Administration. The PSI group is supported by the Swiss Academy of Natural Sciences and the Swiss National Science Foundation (grants 2100049343 and 2000-058827). MSSL acknowledges support from the Particle Physics and Astronomy Research Council. This work is based on observations obtained with XMM-Newton, an ESA science mission with instruments and contributions directly funded by ESA member states and the USA (NASA).

\section{References}

Antunes, A., Nagase, F., \& White, N. E. 1994, ApJ, 436, L83 Arnaud, M., \& Raymond, J. 1992, ApJ, 398, 394

Audard, M., Güdel, M., Drake, J. J., \& Kashyap, V. 2000, ApJ, in press

Audard, M., Güdel, M., \& Mewe, R. 2001, A\&A, 365, L318

Ayres, T. R., Brown, A., Osten, R. A., et al. 2001, ApJ, in press

Bar-Shalom, A., Klapisch, M., Goldstein, W. H., \& Oreg, J. 1998, The HULLAC Code for Atomic Physics, unpublished den Herder, J. W., Brinkman, A. C., Kahn, S. M., et al. 2001, A\&A, 365, L7

Doyle, J. G., \& Butler, J. 1985, Nat, 318, 378

Drake, J. J., Laming, J. M., \& Widing, K. G. 1995, ApJ, 443, 393

Drake, J. J., Laming, J. M., \& Widing, K. G. 1997, ApJ, 478, 403

Drake, S. A. 1996, in 6th Ann. Astrophysics Conference, ASP Conf. Proc., X-ray Measurements of Coronal Abundances, ed. S. S. Holt, \& G. Sonneborn (San Francisco: ASP), 215

Feldman, U. 1992, Phys. Scr., 46, 202

Feldman, U., Mandelbaum, P., Seely, J. F., Doschek, G. A., \& Gursky, H. 1992, ApJS, 81, 387

Feldman, U., \& Widing, K. G. 1990, ApJ, 363, 292

Gabriel, A. H., \& Jordan, C. 1969, MNRAS, 145, 241

Güdel, M. 1994, ApJS, 90, 743

Güdel, M., Linsky, J. L., Brown, A., \& Nagase, F. 1999, ApJ, 511,405

Hénoux J.-C. 1995, Adv. Space Res., 15, 23

Mazzotta, P., Mazzitelli, G., Colafrancesco, S., \& Vittorio, N. 1998, A\&AS, 133, 403

Meyer, J. P. 1985, ApJ, 57, 173

Mewe, R., Kaastra, J. S., van den Oord, G. H. J., Vink, J., \& Tawara, Y. 1997, A\&A, 320, 147

Murphy, R. J., Ramaty, R., Kozlovsky, B., \& Reames, D. V. 1991, ApJ, 371, 793

Pasquini, L., Schmitt, J. H. M. M., \& Pallavicini, R. 1989, A\&A, 226, 225

Randich, S., Giampapa, M. S., \& Pallavicini, R. 1994, A\&A, 283,893

Schmelz, J. T. 1993, ApJ, 408, 373

von Steiger, R., \& Geiss, J. 1989, A\&A, 225, 222

Wang, Y.-M. 1996, ApJ, 464, L91

White, N. E. 1996, in ASP Conf. Ser. 109, Cool stars, Stellar Systems and the Sun, 193 